\title{
Large time behavior for a multidimensional chemotaxis model
}

Lan Luo

\section{"Correspondence:}

lanluogu@sina.com

School of Mathematics and

Information Science, Guangzhou

University, Guangzhou, 510006,

P.R. China

\begin{abstract}
We investigate the global existence and large time behavior of the solutions to the Cauchy problem of a multidimensional chemotaxis model with initial data close to a constant state. We also obtain the time decay rate of $k$ th-order derivatives of these solutions.
\end{abstract}

MSC: $35 \mathrm{G} 25 ; 35 \mathrm{M} 10$

Keywords: chemotaxis model; global existence; large time behavior; time decay rate

\section{Introduction}

In this paper, we consider the Cauchy problem of the multidimensional chemotaxis model

$$
\left\{\begin{array}{l}
\partial_{t} u=\Delta u+\nabla \cdot(u \nabla \ln v), \\
\partial_{t} v=u v-\mu v
\end{array}\right.
$$

where $(t, x) \in \mathbf{R}^{+} \times \mathbf{R}^{n}, u(t, x)$ and $v(t, x)$ denote the cell density and the chemical concentration, respectively, and $\mu$ is a constant.

The system (1.1) was proposed by Othmer and Stevens [1] to describe the chemotactic movement of particles where the chemicals are non-diffusible and can modify the local environment for succeeding passages. For example, myxobacteria produce lime over which their cohorts can move more readily and ants can follow trails left by predecessors [2]. One direct application of (1.1) is to model haptotaxis where cells move toward an increasing concentration of immobilized signals such as surface or matrix-bound adhesive molecules.

For the sake of simplicity, we set $w=\mu t+\ln v$. Therefore we get from (1.1)

$$
\left\{\begin{array}{l}
\partial_{t} u=\Delta u+\nabla \cdot(u \nabla w), \\
\partial_{t} w=u
\end{array}\right.
$$

Letting $\bar{p}>0$ be a constant, we substitute

$$
u=p+\bar{p}, \quad \mathbf{q}=\nabla w
$$

(c) The Author(s) 2017. This article is distributed under the terms of the Creative Commons Attribution 4.0 International License (http://creativecommons.org/licenses/by/4.0/), which permits unrestricted use, distribution, and reproduction in any medium, provided you give appropriate credit to the original author(s) and the source, provide a link to the Creative Commons license, and indicate if changes were made. 
into the system (1.2) to get

$$
\left\{\begin{array}{l}
\partial_{t} p-\bar{p} \operatorname{div} \mathbf{q}-\Delta p=\operatorname{div}(p \mathbf{q}) \\
\partial_{t} \mathbf{q}-\nabla p=0 \\
\nabla \times \mathbf{q}=0
\end{array}\right.
$$

To represent the results in this paper, the following notations are needed. We use $(\cdot, \cdot)$ to denote the standard inner products in $L^{2}\left(\mathbf{R}^{n}\right)$ and $\|\cdot\|$ is the corresponding norm in $L^{2}\left(\mathbf{R}^{n}\right)$. For any $r \in[1, \infty], L^{r}\left(\mathbf{R}^{n}\right)$ is the usual Lebesgue space on $\mathbf{R}^{n}$. We define the operator $\Lambda^{s}$ with $s \in \mathbf{R}$ by

$$
\Lambda^{s} f(x):=\int_{\mathbf{R}^{n}}|\xi|^{s} \hat{f}(\xi) e^{2 \pi i x \cdot \xi} d \xi
$$

where $\hat{f}(\xi)=\mathcal{F} f(\xi)$ is the Fourier transform of $f(x)$. We shall use $D^{k}$ with an integer $k \geq 0$ to stand for the usual spatial derivatives of order $k$. When $k<0$ or $k$ is not a positive integer, $D^{k}$ stands for $\Lambda^{k}$. We then recall the homogeneous Besov spaces. Let $\phi \in C_{c}^{\infty}\left(\mathbf{R}_{\xi}^{n}\right)$ be such that $\phi(\xi)=1$ when $|\xi| \leq 1$ and $\phi(\xi)=0$ when $|\xi| \geq 2$. Let $\varphi(\xi)=\phi(\xi)-\phi(2 \xi)$ and $\varphi_{j}(\xi)=\varphi\left(\frac{\xi}{2^{j}}\right)$ for any $j \in \mathbf{Z}$. Then $\sum_{j \in \mathbf{Z}} \varphi_{j}(\xi)=1$ if $\xi \neq 0$. We define $\Delta_{j}:=\mathcal{F}^{-1}\left(\varphi_{j}\right) * f$, then, for $\varrho \in \mathbf{R}$ and $1 \leq r, s \leq \infty$, we define the homogeneous Besov spaces $\dot{B}_{r, s}^{\varrho}\left(\mathbf{R}^{n}\right)$ with the norm $\|\cdot\|_{\dot{B}_{r, s}}$ defined by

$$
\|f\|_{\dot{B}_{r, s}^{\varrho}}:=\left(\sum_{j \in \mathbf{Z}} 2^{\varrho s j}\left\|\Delta_{j} f\right\|_{L^{r}}^{s}\right)^{\frac{1}{s}}, \quad\|f\|_{\dot{B}_{r, \infty}^{\varrho}}:=\sup _{j \in \mathbf{Z}}\left(2^{\varrho j}\left\|\Delta_{j} f\right\|_{L^{r}}\right) .
$$

We also use $\left\|\Lambda^{k}(p, \mathbf{q})(t)\right\|^{2}:=\left\|\Lambda^{k} p(t)\right\|^{2}+\left\|\Lambda^{k} \mathbf{q}(t)\right\|^{2}$ and denote by $C$ a generic positive constant which varies from line to line. For the energy estimates, the instant energy functional for a solution $(p, \mathbf{q})(t, x)$ to the system (1.4), denoted by $\mathcal{E}_{N}(t)$ has the equivalent relation with some integer $N \geq 0$,

$$
\mathcal{E}_{N}(t) \sim \sum_{k=0}^{N}\left\|D^{k}(p, \mathbf{q})(t)\right\|^{2}
$$

In addition, the corresponding dissipation functional denoted by $\mathcal{D}_{N}(t)$ satisfies

$$
\mathcal{D}_{N}(t) \sim \sum_{k=1}^{N}\left\|D^{k}(p, \mathbf{q})(t)\right\|^{2}+\left\|D^{N+1} p(t)\right\|^{2}
$$

Here the notation $\mathcal{A} \sim \mathcal{B}$ is used to denote that there exist two generic positive constants $C_{1}<C_{2}$ such that $C_{1} \mathcal{B} \leq \mathcal{A} \leq C_{2} \mathcal{B}$.

With the above preparation, the main results in this paper can be stated as follows.

Theorem 1.1 There exists $\epsilon>0$ such that if $\mathcal{E}_{N}(0) \leq \epsilon$ with $N>\frac{n}{2}+1$ and $n \geq 3$, there is a unique global solution $(p, \mathbf{q})=(p, \mathbf{q})(t, x)$ to the system $(1.4)$ with $(p, \mathbf{q})(0, x)=\left(p_{0}(x), \mathbf{q}_{0}(x)\right)$ that satisfies

$$
\frac{d}{d t} \mathcal{E}_{N}(t)+C \mathcal{D}_{N}(t) \leq 0 .
$$


This global solution has the following time decay:

$$
\left\|\Lambda^{\ell}(p, \mathbf{q})(t)\right\|^{2} \leq C \epsilon(1+t)^{-\ell} \quad \text { for any } \ell \in[0, N] .
$$

If we further assume $\left\|\left(p_{0}, \mathbf{q}_{0}\right)\right\|_{\dot{B}_{2, \infty}^{-}}<\infty$ with $\varrho \in\left(0, \frac{n}{2}\right]$, we have

$$
\left\|\Lambda^{\ell}(p, \mathbf{q})(t)\right\|^{2} \leq C(1+t)^{-(\ell+\varrho)} \quad \text { for any } \ell \in[-\varrho, N-1]
$$

Remark 1.2 Similar time decay rates to (1.8) and (1.9) were obtained when the initial data in the additional negative homogeneous Sobolev space $\dot{H}^{-} \varrho\left(\mathbf{R}^{n}\right)$ with $\varrho \in\left(0, \frac{n}{2}\right)$ for equation (1.4) with some perturbation term in [3] the referee told us. Compared to the results in [3], our results can include the endpoint cases while the equation considered in [3] is a little more complicated. In some sense our results generalize their partial time decay rates results and we believe that our proof methods can also apply to the equation considered in [3] to obtain the time decay rates of the endpoints. In addition, the other small assumptions of the initial data are imposed in $[4,5]$, some time decay rates results can be obtained and our results also improve the time decay rate results in $[4,5]$. As to the chemotaxis significance of the time decay rates of equation (1.4), we refer the reader to [3-5], where the authors give more discussions.

From Theorem 1.1, (1.2) and (1.3), we have the following results on the asymptotic behavior of solutions to the system (1.1).

Corollary 1.3 There exists $\epsilon>0$ such that if $\sum_{k=0}^{N}\left\|D^{k}\left(u_{0}-\bar{p}, \nabla \ln v_{0}\right)\right\|^{2} \leq \epsilon$ with $N>$ $\frac{n}{2}+1$ and $n \geq 3$, then the Cauchy problem (1.1) has a global solution $(u, v)=(u, v)(t, x)$ with $(u, v)(0, x)=\left(u_{0}(x), v_{0}(x)\right)$ satisfying for all $t>0$

$$
\sum_{k=0}^{N}\left\|D^{k}(u-\bar{p}, \nabla \ln v)(t)\right\|^{2} \leq C \epsilon
$$

This global solution has the following time decay:

$$
\left\|\Lambda^{\ell}(u-\bar{p}, \nabla \ln v)(t)\right\|^{2} \leq C \epsilon(1+t)^{-\ell} \quad \text { for any } \ell \in[0, N] .
$$

If we further assume $\left\|\left(u_{0}-\bar{p}, \nabla \ln v_{0}\right)\right\|_{\dot{B}_{2, \infty}^{-}}<\infty$ with $\varrho \in\left(0, \frac{n}{2}\right]$, we have

$$
\left\|\Lambda^{\ell}(u-\bar{p}, \nabla \ln v)(t)\right\|^{2} \leq C(1+t)^{-(\ell+\varrho)} \quad \text { for any } \ell \in[-\varrho, N-1]
$$

Let us review some work related to the study in this paper. The one-dimensional version of the system (1.1) has been studied for both Cauchy problems and initial-boundary value problems, for instance, in $[6,7]$ for bounded domains and in $[8,9]$ for nonlinear stabilities of traveling waves. However, there are few results for the multidimensional cases of the system (1.1). The initial-boundary value problems are studied in [10,11]. The local and global existence of the solution to the Cauchy problem near some constant state is obtained in [5]. Hao [12] proved existence and uniqueness of global solutions for initial data close to some constant state in critical Besov space with minimal regularity. The proof 
was in the Chemin-Lerner space framework (see e.g. [13, 14]). In [4], the authors obtained the global weak existence and partial time decay of the solutions to the system (1.4) near some constant state in the slightly low regular Sobolev space. It was studied in [1] and a comprehensive qualitative and numerical analysis were provided. We will not attempt to exhaust references in this paper. We refer reader to $[1,3-6,10-12,15]$ and the references therein for more discussions in this direction.

In this paper we consider precise large time behavior of the global solutions to the system (1.1) near some constant state, motivated by $[4,5,12]$. We first construct the uniform estimates, which are used to obtain global existence and time decay rate under the assumption of initial energy being small enough. Once a global solution are constructed, by using a time-weighted methods, we can prove the time decay rate of the $k$ th-order derivatives of these solutions approaching a constant state at a time decay rate $O\left(t^{-\frac{k}{2}}\right)$ without additional assumption. We remark that the time-weighted methods was also applied to prove the time decay rate for the fluid system $[16,17]$. If we further assume that the initial data is in the negative Besov space (not necessary small) by a similar interpolation to [18, 19], we can obtain the fast time decay rate.

\section{Some basic estimates}

In this section we first represent some elementary inequalities and then we use these results to handle the nonlinear term.

Lemma 2.1 ([20]) For any $f, g \in L^{\infty}\left(\mathbf{R}^{n}\right) \cap H^{k}\left(\mathbf{R}^{n}\right)$, we have

$$
\left\|D^{k}(f g)\right\| \leq C\left(\|f\|_{L^{\infty}}\left\|D^{k} g\right\|+\|g\|_{L^{\infty}}\left\|D^{k} f\right\|\right)
$$

Lemma $2.2([18])$ Suppose that $m \neq \varrho$. We have the interpolation estimate

$$
\left\|\Lambda^{k} g\right\|_{L^{r}} \leq C\left\|\Lambda^{m} g\right\|_{L^{2}}^{1-\theta}\|g\|_{\dot{B}_{2, \infty}^{\varrho}}^{\theta} \leq C\left\|\Lambda^{m} g\right\|_{L^{2}}^{1-\theta}\left\|\Lambda^{\varrho} g\right\|_{L^{2}}^{\theta},
$$

where $0<\theta<1$ and $2 \leq r \leq \infty$ and

$$
k+\frac{n}{2}-\frac{n}{r}=m(1-\theta)+\varrho \theta .
$$

Lemma 2.3 If $0 \leq k \leq N-1$, it follows that

$$
\left|\left(D^{k} \operatorname{div}(p \mathbf{q}), \operatorname{div} D^{k} \mathbf{q}\right)\right| \leq C \sqrt{\mathcal{E}_{N}(t)}\left\|D^{k+1}(p, \mathbf{q})\right\|^{2}
$$

and

$$
\left|\left(D^{k} \operatorname{div}(p \mathbf{q}), D^{k} p\right)\right| \leq C \sqrt{\mathcal{E}_{N}(t)} \sum_{m=k+1}^{N}\left\|D^{m}(p, \mathbf{q})\right\|^{2} .
$$

We have

$$
\left|\left(D^{N} \operatorname{div}(p \mathbf{q}), D^{N} p\right)\right| \leq C\|(p, \mathbf{q})\|_{L^{\infty}}\left\|D^{N}(p, \mathbf{q})\right\|\left\|D^{N+1} p\right\| .
$$


Proof By (1.5) and Lemma 2.2, we have, for $\theta=\left(\frac{n}{2}-1\right) /\left[\frac{n}{2}\right]$,

$$
\|p\|_{L^{\infty}}^{2} \leq C\|D p\|^{2(1-\theta)}\left\|D^{\left[\frac{n}{2}\right]+1} p\right\|^{2 \theta} \leq C \mathcal{E}_{N}(t)
$$

It follows from this and (2.1) that

$$
\left|\left(D^{k} \operatorname{div}(p \mathbf{q}), \operatorname{div} D^{k} \mathbf{q}\right)\right| \leq C\|(p, \mathbf{q})\|_{L^{\infty}}\left\|D^{k+1}(p, \mathbf{q})\right\|^{2} \leq C \sqrt{\mathcal{E}_{N}(t)}\left\|D^{k+1}(p, \mathbf{q})\right\|^{2}
$$

It follows from (2.1) that

$$
\begin{aligned}
\left|\left(D^{k} \operatorname{div}(p \mathbf{q}), D^{k} p\right)\right| & =\left|\left(D^{k}(p \mathbf{q}), D^{k+1} p\right)\right| \\
& \leq C\|(p, \mathbf{q})\|_{L^{\infty}}\left\|D^{k}(p, \mathbf{q})\right\|\left\|D^{k+1} p\right\| .
\end{aligned}
$$

Then we see from (2.8) that (2.6) holds. Next we prove (2.5). If $k=0,(2.5)$ follows from (2.7) and (2.8). If $1 \leq k \leq \frac{n}{2}$, it follows from (2.2) that

$$
\|p\|_{L^{\infty}}\left\|\Lambda^{k} p\right\| \leq C\left\|\Lambda^{\alpha} p\right\|^{\frac{k}{k+1}}\left\|\Lambda^{\left[\frac{n}{2}\right]+1} p\right\|^{\frac{1}{k+1}}\left\|\Lambda^{k+1} p\right\|^{\frac{k}{k+1}}\|p\|^{\frac{1}{k+1}}
$$

where $\alpha=\frac{n}{2}-\left(\left[\frac{n}{2}\right]+1-\frac{n}{2}\right) \frac{1}{k} \in\left[0, \frac{n}{2}\right)$.

If $\alpha \in \mathbf{Z}$, by (2.8) and (2.9), we get (2.5). If $\alpha \notin \mathbf{Z}$, it follows from (2.2) that

$$
\left\|\Lambda^{\alpha} p\right\| \leq\left\|\Lambda^{[\alpha]} p\right\|^{[\alpha]+1-\alpha}\left\|\Lambda^{[\alpha]+1} p\right\|^{\alpha-[\alpha]} .
$$

By this, (2.8) and (2.9), we obtain (2.5). If $\frac{n}{2}<k \leq N-1$, we have from (2.2)

$$
\|p\|_{L^{\infty}}\left\|\Lambda^{k} p\right\| \leq C\|p\|^{1-\theta}\left\|\Lambda^{k+1} p\right\|^{\theta}\left\|\Lambda^{k+1} p\right\|^{1-\theta}\left\|\Lambda^{\alpha} p\right\|^{\theta}
$$

where $\theta=\frac{n}{2(k+1)}$ and $\alpha=\left(1-\frac{2}{n}\right)(k+1) \in(0, k+1)$. If $\alpha \in \mathbf{Z}$, by (2.8) and (2.11), we get (2.5). If $\alpha \notin \mathbf{Z}$, we use (2.10), (2.11) and (2.8) to obtain (2.5).

\section{Energy analysis}

In this section we first deduce the uniform estimates of the system (1.4) and make use of the time-weighted methods to the time decay rate (1.7). Then we use the properties of the Besov space and the interpolation to get the fast time decay rate.

Theorem 3.1 Letting $(p, \mathbf{q})=(p, \mathbf{q})(t, x)$ be the solution to the system $(1.4)$ satisfying $\mathcal{E}_{N}(t)<$ $\epsilon$, we have the estimate

$$
\frac{d}{d t} \mathcal{E}_{N}(t)+C \mathcal{D}_{N}(t) \leq 0
$$

Here $\mathcal{E}_{N}(t)$ and $\mathcal{D}_{N}(t)$ are defined as (1.5) and (1.6).

Proof Taking the derivative $D^{k}$ of (1.4) with $0 \leq k \leq N$, we can obtain

$$
\frac{1}{2} \frac{d}{d t}\left(\left\|D^{k} p\right\|^{2}+\bar{p}\left\|D^{k} \mathbf{q}\right\|^{2}\right)+\left\|\nabla D^{k} p\right\|^{2}=\left(D^{k} \operatorname{div}(p \mathbf{q}), D^{k} p\right)
$$


For the system (1.4), we have for $0 \leq k \leq N-1$

$$
\begin{aligned}
& \frac{1}{2} \frac{d}{d t}\left\|\operatorname{div} D^{k} \mathbf{q}\right\|^{2}-\frac{d}{d t}\left(D^{k} p, \operatorname{div} D^{k} \mathbf{q}\right)+\bar{p}\left\|\operatorname{div} D^{k} \mathbf{q}\right\|^{2}-\left\|\nabla D^{k} p\right\|^{2} \\
& \quad=-\left(D^{k} \operatorname{div}(p \mathbf{q}), \operatorname{div} D^{k} \mathbf{q}\right) .
\end{aligned}
$$

For any $\kappa>0$ small enough, we have from (3.2) and (3.3)

$$
\begin{aligned}
\frac{d}{d t}\left\{\sum_{k=0}^{N}\left(\left\|D^{k} p\right\|^{2}+\bar{p}\left\|D^{k} \mathbf{q}\right\|^{2}\right)+\kappa \sum_{k=0}^{N-1}\left(\left\|\operatorname{div} D^{k} \mathbf{q}\right\|^{2}-2\left(D^{k} p, \operatorname{div} D^{k} \mathbf{q}\right)\right)\right\} \\
\quad+\sum_{k=0}^{N-1}\left\{2(1-\kappa)\left\|\nabla D^{k} p\right\|^{2}+2 \kappa \bar{p}\left\|\operatorname{div} D^{k} \mathbf{q}\right\|^{2}\right\}+2\left\|D^{N+1} p\right\|^{2} \\
=2 \sum_{k=0}^{N}\left(D^{k} \operatorname{div}(p \mathbf{q}), D^{k} p\right)-2 \kappa \sum_{k=0}^{N-1}\left(D^{k} \operatorname{div}(p \mathbf{q}), \operatorname{div} D^{k} \mathbf{q}\right) .
\end{aligned}
$$

Notice that the equality $\left\|\nabla_{x} \mathbf{q}\right\|^{2}=\|\operatorname{div} \mathbf{q}\|^{2}+\|\nabla \times \mathbf{q}\|^{2}$ holds on p.2015 of [21]. By the fact that $\nabla \times \mathbf{q}=0$, we have $\left\|\operatorname{div} D^{k} \mathbf{q}\right\|^{2}=\left\|D^{k+1} \mathbf{q}\right\|^{2}$. By this, for any $\kappa>0$ small enough, one has

$$
\begin{aligned}
\mathcal{E}_{N}(t) & =\sum_{k=0}^{N}\left(\left\|D^{k} p\right\|^{2}+\bar{p}\left\|D^{k} \mathbf{q}\right\|^{2}\right)+\kappa \sum_{k=0}^{N-1}\left(\left\|\operatorname{div} D^{k} \mathbf{q}\right\|^{2}-2\left(D^{k} p, \operatorname{div} D^{k} \mathbf{q}\right)\right) \\
& \sim \sum_{k=0}^{N}\left\|D^{k}(p, \mathbf{q})\right\|^{2}, \\
\mathcal{D}_{N}(t) & =\sum_{k=0}^{N-1}\left\{2(1-\kappa)\left\|\nabla D^{k} p\right\|^{2}+2 \kappa \bar{p}\left\|\operatorname{div} D^{k} \mathbf{q}\right\|^{2}\right\}+2\left\|D^{N+1} p\right\|^{2} \\
& \sim \sum_{k=1}^{N}\left\|D^{k}(p, \mathbf{q})\right\|^{2}+\left\|D^{N+1} p\right\|^{2} .
\end{aligned}
$$

By Lemma 2.3 we have

$$
\left|\sum_{k=0}^{N}\left(D^{k} \operatorname{div}(p \mathbf{q}), D^{k} p\right)\right|+\left|\sum_{k=0}^{N-1}\left(D^{k} \operatorname{div}(p \mathbf{q}), \operatorname{div} D^{k} \mathbf{q}\right)\right| \leq C \sqrt{\mathcal{E}_{N}(t)} \mathcal{D}_{N}(t)
$$

Since $\mathcal{E}_{N}(t)<\epsilon$, by these, we see from (3.4) that (3.1) holds.

Remark 3.2 By Theorem 3.1 we have derived a priori estimates. Based on this uniform estimate the global existence is proved by the standard continuation argument together with the local in time existence theory; for example, see [5].

In order to show the time decay of the solutions obtained above, we need the refined energy estimates of each order derivatives of the solutions to the system (1.4). For this purpose, we shall prove the following lemma. 
Lemma 3.3 Assume that $(p, \mathbf{q})=(p, \mathbf{q})(t, x)$ be the solution to the system (1.4) satisfying $\mathcal{E}_{N}(t)<\epsilon$. For any fixed $m \in\{0,1, \ldots, N-1\}$, we have the following estimate:

$$
\frac{d}{d t} \mathcal{E}^{m}(t)+C \mathcal{D}^{m}(t) \leq 0
$$

Here $\mathcal{E}^{m}(t)$ and $\mathcal{D}^{m}(t)$ are defined by

$$
\begin{aligned}
& \mathcal{E}^{m}(t)=\sum_{k=m}^{N}\left(\left\|D^{k} p\right\|^{2}+\bar{p}\left\|D^{k} \mathbf{q}\right\|^{2}\right)+\kappa \sum_{k=m}^{N-1}\left(\left\|\operatorname{div} D^{k} \mathbf{q}\right\|^{2}-2\left(D^{k} p, \operatorname{div} D^{k} \mathbf{q}\right)\right) \\
& \mathcal{D}^{m}(t)=\sum_{k=m}^{N-1}\left\{2(1-\kappa)\left\|\nabla D^{k} p\right\|^{2}+2 \kappa \bar{p}\left\|\operatorname{div} D^{k} \mathbf{q}\right\|^{2}\right\}+\left\|D^{N+1} p\right\|^{2}
\end{aligned}
$$

where $\kappa>0$ is small enough.

Proof For any $\kappa>0$ small enough, we have from (3.2) and (3.3)

$$
\begin{aligned}
& \frac{d}{d t}\left\{\sum_{k=m}^{N}\left(\left\|D^{k} p\right\|^{2}+\bar{p}\left\|D^{k} \mathbf{q}\right\|^{2}\right)+\kappa \sum_{k=m}^{N-1}\left(\left\|\operatorname{div} D^{k} \mathbf{q}\right\|^{2}-2\left(D^{k} p, \operatorname{div} D^{k} \mathbf{q}\right)\right)\right\} \\
& \quad+\sum_{k=m}^{N-1}\left\{2(1-\kappa)\left\|\nabla D^{k} p\right\|^{2}+2 \kappa \bar{p}\left\|\operatorname{div} D^{k} \mathbf{q}\right\|^{2}\right\}+2\left\|D^{N+1} p\right\|^{2} \\
& =2 \sum_{k=m}^{N}\left(D^{k} \operatorname{div}(p \mathbf{q}), D^{k} p\right)-2 \kappa \sum_{k=m}^{N-1}\left(D^{k} \operatorname{div}(p \mathbf{q}), \operatorname{div} D^{k} \mathbf{q}\right) .
\end{aligned}
$$

Since $\left\|\operatorname{div} D^{k} \mathbf{q}\right\|^{2}=\left\|D^{k+1} \mathbf{q}\right\|^{2}$ and $\kappa>0$ is small enough, one has from (3.6) and (3.7) that

$$
\mathcal{E}^{m}(t) \sim \sum_{k=m}^{N}\left\|D^{k}(p, \mathbf{q})\right\|^{2}, \quad \text { and } \quad \mathcal{D}^{m}(t) \sim \sum_{k=m+1}^{N}\left\|D^{k}(p, \mathbf{q})\right\|^{2}+\left\|D^{N+1} p\right\|^{2}
$$

By Lemma 2.3 we have

$$
\left|\sum_{k=m}^{N}\left(D^{k} \operatorname{div}(p \mathbf{q}), D^{k} p\right)\right|+\left|\sum_{k=m}^{N-1}\left(D^{k} \operatorname{div}(p \mathbf{q}), \operatorname{div} D^{k} \mathbf{q}\right)\right| \leq C \sqrt{\mathcal{E}_{N}(t)} \mathcal{D}^{m}(t) .
$$

Since $\mathcal{E}_{N}(t)<\epsilon$, by these, we see from (3.8) that (3.5) holds.

In the following we will use the time-weighted methods to show the time decay of the solutions to the system (1.4).

Theorem 3.4 Assume that $(p, \mathbf{q})=(p, \mathbf{q})(t, x)$ be the solution to the system (1.4) satisfying $\mathcal{E}_{N}(t)<\epsilon$. We have the time decay estimate, for any $m \in[0, N]$,

$$
\left\|\Lambda^{m}(p, \mathbf{q})\right\|^{2} \leq C \epsilon(1+t)^{-m}
$$


Proof For any $m \in\{1, \ldots, N-1\}$, we see from (3.5) that

$$
\frac{d}{d t}\left\{(1+t)^{m} \mathcal{E}^{m}(t)\right\}-m(1+t)^{m-1} \mathcal{E}^{m}(t)+(1+t)^{m} \mathcal{D}^{m}(t) \leq 0 .
$$

Letting the constants $C_{m}>0$ and $C_{m} \gg C_{m+1}$ be large enough, we define the functional

$$
E(t)=\sum_{m=0}^{N-1} C_{m}(1+t)^{m} \mathcal{E}^{m}(t) .
$$

By (3.10), (3.11) and (3.5) with $m=0$, we see that

$$
E(t)+C \sum_{m=0}^{N-1} \int_{0}^{t}(1+s)^{m} \mathcal{D}^{m}(s) d s \leq E(0)
$$

Since $\mathcal{E}_{N}(t)<\epsilon$, for any $m \in\{0,1, \ldots, N-1\}$, we have from (3.12) and (3.9)

$$
\left\|D^{m}(p, \mathbf{q})\right\|^{2} \leq C \mathcal{E}^{m}(t) \leq C \epsilon(1+t)^{-m} .
$$

By (3.2) and (2.6) one has

$$
\begin{aligned}
& \frac{1}{2} \frac{d}{d t}\left(\left\|D^{N} p\right\|^{2}+\bar{p}\left\|D^{N} \mathbf{q}\right\|^{2}\right)+C\left\|\nabla D^{N} p\right\|^{2} \\
& \quad \leq C_{\varepsilon}\|(p, \mathbf{q})\|_{L^{\infty}}^{2}\left\|D^{N}(p, \mathbf{q})\right\|^{2}+\varepsilon\left\|\nabla D^{N} p\right\|^{2} .
\end{aligned}
$$

Taking $\varepsilon>0$ small enough, we have

$$
\frac{1}{2} \frac{d}{d t}\left(\left\|D^{N} p\right\|^{2}+\bar{p}\left\|D^{N} \mathbf{q}\right\|^{2}\right)+C\left\|\nabla D^{N} p\right\|^{2} \leq C_{\varepsilon}\|(p, \mathbf{q})\|_{L^{\infty}}^{2}\left\|D^{N}(p, \mathbf{q})\right\|^{2} .
$$

It follows from (3.14) that

$$
\begin{aligned}
& \frac{d}{d t}\left\{(1+t)^{N}\left(\left\|D^{N} p\right\|^{2}+\bar{p}\left\|D^{N} \mathbf{q}\right\|^{2}\right)\right\}-C N(1+t)^{N-1}\left\|D^{N}(p, \mathbf{q})\right\|^{2} \\
& \quad \leq C(1+t)^{N}\|(p, \mathbf{q})\|_{L^{\infty}}^{2}\left\|D^{N}(p, \mathbf{q})\right\|^{2} .
\end{aligned}
$$

By (3.13) and (2.7), one has $\|(p, \mathbf{q})\|_{L^{\infty}}^{2} \leq C \epsilon(1+t)^{-1}$. It follows from (3.15) that

$$
(1+t)^{N}\left\|D^{N}(p, \mathbf{q})\right\|^{2} \leq C \int_{0}^{t}(1+s)^{N-1}\left\|D^{N}(p, \mathbf{q})(s)\right\|^{2} d s+C\left\|D^{N}\left(p_{0}, \mathbf{q}_{0}\right)\right\|^{2} .
$$

Since $\mathcal{E}_{N}(t)<\epsilon$, we have from (3.12) and the definition of $\mathcal{D}^{N-1}(s)$ that

$$
C \int_{0}^{t}(1+s)^{N-1}\left\|D^{N}(p, \mathbf{q})(s)\right\|^{2} d s+C\left\|D^{N}\left(p_{0}, \mathbf{q}_{0}\right)\right\|^{2} \leq C \epsilon .
$$

By the above estimates one has

$$
\left\|D^{N}(p, \mathbf{q})\right\|^{2} \leq C \epsilon(1+t)^{-N} .
$$

By this, (3.13) and Lemma 2.2, we can obtain the desired estimates (1.8). 
To show the fast time decay under the additional assumption, for this, we need to show the propagation of the solutions in $\dot{B}_{2, \infty}^{-}$.

Lemma 3.5 For any $\varrho \in\left(0, \frac{n}{2}\right]$, for the system (1.4), we have

$$
\frac{d}{d t}\left(\|p\|_{\dot{B}_{2, \infty}^{-Q}}^{2}+\bar{p}\|\mathbf{q}\|_{\dot{B}_{2, \infty}^{-e}}^{2}\right)+C\|\nabla p\|_{\dot{B}_{2, \infty}^{-}}^{2} \leq C\|p\|_{L_{x}^{\frac{n}{\varrho}}}^{2}\|\mathbf{q}\|^{2} .
$$

Proof Taking the operation $\Delta_{j}$ on the sides of (1.4), we have

$$
\frac{1}{2} \frac{d}{d t}\left\|\Delta_{j} p\right\|^{2}-\bar{p}\left(\Delta_{j} \operatorname{div} \mathbf{q}, \Delta_{j} p\right)+\left\|\nabla \Delta_{j} p\right\|^{2}=\left(\operatorname{div} \Delta_{j}(p \mathbf{q}), \Delta_{j} p\right)
$$

and

$$
\frac{1}{2} \frac{d}{d t}\left\|\Delta_{j} \mathbf{q}\right\|^{2}-\left(\nabla \Delta_{j} p, \Delta_{j} \mathbf{q}\right)=0
$$

By these we have

$$
\frac{1}{2} \frac{d}{d t}\left(\left\|\Delta_{j} p\right\|^{2}+\bar{p}\left\|\Delta_{j} \mathbf{q}\right\|^{2}\right)+\left\|\nabla \Delta_{j} p\right\|^{2}=\left(\operatorname{div} \Delta_{j}(p \mathbf{q}), \Delta_{j} p\right)
$$

We have

$$
\begin{aligned}
\left|\left(\operatorname{div} \Delta_{j}(p \mathbf{q}), \Delta_{j} p\right)\right| & =\left|\left(\Delta_{j}(p \mathbf{q}), \nabla \Delta_{j} p\right)\right| \leq 2^{\varrho j} C\|p \mathbf{q}\|_{L^{r}}\left\|\nabla \Delta_{j} p\right\| \\
& \leq 2^{\rho j} C_{\varepsilon}\|p\|_{L_{x}^{\frac{n}{\varrho}}}^{2}\|\mathbf{q}\|^{2}+\varepsilon\left\|\nabla \Delta_{j} p\right\|^{2} .
\end{aligned}
$$

Here we have used the Bernstein type inequality in [14] for $\varrho=\frac{n}{r}-\frac{n}{2} \in\left(0, \frac{n}{2}\right]$ and $r \in[1,2)$. By using the definition of $\dot{B}_{2, \infty}^{-}$and taking $\varepsilon>0$ small enough, it follows from (3.17) that (3.16) holds.

To finish the proof of the fast time decay we will make use of an interpolation method similar to $[18,19]$.

Proof of (1.9) in Theorem 1.1 By (3.16) we have, for any $\varrho \in\left(0, \frac{n}{2}\right]$,

$$
\|(p, \mathbf{q})(t)\|_{\dot{B}_{2, \infty}^{-}}^{2} \leq C\left\|\left(p_{0}, \mathbf{q}_{0}\right)\right\|_{\dot{B}_{2, \infty}^{-\varrho}}^{2}+C \int_{0}^{t}\|p\|_{L_{x}^{\frac{n}{\varrho}}}^{2}\|\mathbf{q}\|^{2} d s
$$

For $\varrho \in\left(0, \frac{n-2}{2}\right]$, we have $1 \leq\left[\frac{n}{2}-\varrho\right]<\frac{n}{2}$ and

$$
\|p\|_{L_{x}^{\frac{n}{\varrho}}}^{2}\|\mathbf{q}\|^{2} \leq C\left\|D^{\left[\frac{n}{2}-\varrho\right]} p\right\|^{2(1-\theta)}\left\|D^{\left[\frac{n}{2}-\varrho\right]+1} p\right\|^{2 \theta}\|\mathbf{q}\|^{2} \leq C \mathcal{E}_{N}(s) \mathcal{D}_{N}(s) .
$$

Here $\theta=\frac{n}{2}-\varrho-\left[\frac{n}{2}-\varrho\right] \in(0,1)$ for $\frac{n}{2}-\varrho \notin \mathbf{Z}$ by Lemma 2.2 and $\theta=0$ for $\frac{n}{2}-\varrho \in \mathbf{Z}$. If $\mathcal{E}_{N}(0) \leq \epsilon$, it follows from (3.1) that for any $t>0$

$$
\mathcal{E}_{N}(t)+C \int_{0}^{t} \mathcal{D}_{N}(s) d s \leq \mathcal{E}_{N}(0) \leq \epsilon
$$


The above three inequalities imply that if $\left\|\left(p_{0}, \mathbf{q}_{0}\right)\right\|_{\dot{B}_{2, \infty}^{-e}} \leq C_{0}$

$$
\|(p, \mathbf{q})(t)\|_{\dot{B}_{2, \infty}^{-e}}^{2} \leq C\left\|\left(p_{0}, \mathbf{q}_{0}\right)\right\|_{\dot{B}_{2, \infty}^{-e}}^{2}+\epsilon^{2} \leq C .
$$

For fixed $m \in\{0,1,2, \ldots, N-1\}$ we have from (2.2)

$$
\left\|D^{m}(p, \mathbf{q})\right\| \leq C\left\|D^{m+1}(p, \mathbf{q})\right\|^{\frac{\varrho+m}{\varrho+m+1}}\|(p, \mathbf{q})\|_{\dot{B}_{2, \infty}^{-\varrho}}^{\frac{1}{\varrho+m+1}} \leq C\left\|D^{m+1}(p, \mathbf{q})\right\|^{\frac{\varrho+m}{\varrho+m+1}} .
$$

It follows from this, (3.6), (3.7) and (3.9) that

$$
\begin{aligned}
\left(\mathcal{E}^{m}(t)\right)^{1+\frac{1}{\varrho+m}} & \leq C\left(\sum_{k=m}^{N}\left\|D^{k}(p, \mathbf{q})\right\|^{2}\right)^{1+\frac{1}{\varrho+m}} \\
& \leq C\left(\sum_{k=m}^{N-1}\left\|D^{k}(p, \mathbf{q})\right\|^{2}\right)^{1+\frac{1}{\varrho+m}}+C\left\|D^{N}(p, \mathbf{q})\right\|^{2\left(1+\frac{1}{\varrho+m}\right)} \\
& \leq C \sum_{k=m}^{N}\left\|D^{k}(p, \mathbf{q})\right\|^{2}+C\left\|D^{N}(p, \mathbf{q})\right\|^{2} \leq C \mathcal{D}^{m}(t) .
\end{aligned}
$$

We have from (3.5) and the above inequality, for $m \in\{0,1,2, \ldots, N-1\}$,

$$
\frac{d}{d t} \mathcal{E}^{m}(t)+C\left(\mathcal{E}^{m}(t)\right)^{1+\frac{1}{\varrho+m}} \leq 0
$$

By using (3.9), we solve this inequality to obtain

$$
\sum_{k=m}^{N}\left\|D^{k}(p, \mathbf{q})\right\|^{2} \leq C(1+t)^{-(m+\varrho)} .
$$

By using Lemma 2.2, we can obtain (1.9) for $\varrho \in\left(0, \frac{n-2}{2}\right]$.

Next we consider the case $\varrho \in\left(\frac{n-2}{2}, \frac{n}{2}\right)$ and $\left\|\left(p_{0}, \mathbf{q}_{0}\right)\right\|_{\dot{B}_{2, \infty}^{-}}<\infty$. In this case, we have $\left\|\left(p_{0}, \mathbf{q}_{0}\right)\right\|_{\dot{B}_{2, \infty}^{-\varrho^{\prime}}}<\infty$ for any $\varrho^{\prime} \in(0, \varrho)$ by interpolation:

$$
\begin{aligned}
\left\|\left(p_{0}, \mathbf{q}_{0}\right)\right\|_{\dot{B}_{2, \infty}^{-\varrho^{\prime}}} & \leq C\left\|\left(p_{0}, \mathbf{q}_{0}\right)\right\|_{\dot{B}_{2, \infty}^{-}-\varrho}^{\varrho^{\prime}}\left\|\left(p_{0}, \mathbf{q}_{0}\right)\right\|_{\dot{B}_{2, \infty}^{0}}^{\left(\varrho-\varrho^{\prime}\right) / \varrho} \\
& \leq C\left\|\left(p_{0}, \mathbf{q}_{0}\right)\right\|_{\dot{B}_{2, \infty}^{-\varrho} / \varrho}^{\varrho^{\prime}}\left\|\left(p_{0}, \mathbf{q}_{0}\right)\right\|^{\left(\varrho-\varrho^{\prime}\right) / \varrho}<\infty .
\end{aligned}
$$

Then (3.22) holds for any $\varrho^{\prime} \in\left(0, \frac{n-2}{2}\right]$ and any $m \in\{0,1,2, \ldots, N-1\}$. We set $\varrho^{\prime}=\frac{n-2}{2}$ with $n \geq 3$. By (3.19) and (3.22) we have

$$
\begin{aligned}
\|(p, \mathbf{q})(s)\|_{L_{x}^{\frac{n}{\varrho}}}^{2}\|(p, \mathbf{q})(s)\|^{2} & \leq C\left\|D^{\left[\frac{n}{2}-\varrho\right]}(p, \mathbf{q})\right\|^{2(1-\theta)}\left\|D^{\left[\frac{n}{2}-\varrho\right]+1}(p, \mathbf{q})\right\|^{2 \theta}\|(p, \mathbf{q})(s)\|^{2} \\
& \leq C(1+s)^{-(1-\theta)\left(\left[\frac{n}{2}-\varrho\right]+\varrho^{\prime}\right)}(1+s)^{-\theta\left(\left[\frac{n}{2}-\varrho\right]+1+\varrho^{\prime}\right)}(1+s)^{-\varrho^{\prime}} \\
& \leq C(1+s)^{-\left(\frac{n}{2}-\varrho+n-2\right)} .
\end{aligned}
$$


Since $\varrho \in\left(\frac{n-2}{2}, \frac{n}{2}\right)$ and $n \geq 3$, one has $\frac{n}{2}-\varrho+n-2>1$. It follows from (3.18) that $\|(p, \mathbf{q})(t)\|_{\dot{B}_{2, \infty}^{-}}<\infty$ for any $t \geq 0$. By this we may repeat the arguments leading to (3.22) and prove that (3.22) holds for the case $\varrho \in\left(\frac{n-2}{2}, \frac{n}{2}\right)$.

Finally if $\varrho=\frac{n}{2}$ and $\left\|\left(p_{0}, \mathbf{q}_{0}\right)\right\|_{\dot{B}_{2, \infty}^{-}}<\infty$, it follows from (3.23) that $\left\|\left(p_{0}, \mathbf{q}_{0}\right)\right\|_{\dot{B}_{2, \infty}^{-\varrho^{\prime}}}<\infty$ for any $\varrho^{\prime} \in\left(0, \frac{n}{2}\right)$. Assume that $\varrho^{\prime}=\frac{n-\delta}{2}$ with $n \geq 3$ and $\delta>0$ small enough such that

$$
\|(p, \mathbf{q})(s)\|_{L_{x}^{\frac{n}{\varrho}}}^{2}\|(p, \mathbf{q})(s)\|^{2}=\|(p, \mathbf{q})(s)\|^{4} \leq C(1+s)^{-(n-\delta)} \leq C(1+s)^{-3 / 2} .
$$

By this we see from (3.18) that $\|(p, \mathbf{q})(t)\|_{\dot{B}_{2, \infty}^{-\varrho}}<\infty$. By similar arguments to (3.22), we can obtain (3.22) for the case $\varrho=\frac{n}{2}$. By using Lemma 2.2, we can obtain (1.9) for any $\varrho \in\left(0, \frac{n}{2}\right]$. This completes the proof of Theorem 1.1.

\section{Competing interests}

The author declares that she has no competing interests.

\section{Acknowledgements}

We would like to thank the referees for their helpful comments and pointing us out $[3,15]$. The research of the author was supported by the NNSFC Grant 11371151.

\section{Publisher's Note}

Springer Nature remains neutral with regard to jurisdictional claims in published maps and institutional affiliations.

Received: 16 January 2017 Accepted: 14 March 2017 Published online: 23 March 2017

\section{References}

1. Othmer, H, Stevens, A: Aggregation, blowup and collapse: the ABC's of taxis in reinforced random walks. SIAM J. Appl. Math. 57, 1044-1081 (1997)

2. Eisenbach, M: Chemotaxis. Imperial College Press, London (2004)

3. Wang, Z-A, Xiang, Z-Y, Yu, P: Asymptotic dynamics on a singular chemotaxis system modeling onset of tumor angiogenesis. J. Differ. Equ. 260(3), 2225-2258 (2016)

4. Deng, C, Li, T: Well-posedness of a 3D parabolic-hyperbolic Keller-Segel system in the Sobolev space framework. J. Differ. Equ. 257(5), 1311-1332 (2014)

5. Li, D, Li, T, Zhao, K: On a hyperbolic-parabolic system modeling chemotaxis. Math. Models Methods Appl. Sci. 21(8), $1631-1650$ (2011)

6. Li, T, Pan, R-H, Zhao, K: Global dynamics of a hyperbolic-parabolic model arising from chemotaxis. SIAM J. Appl. Math. 72(1), 417-443 (2012)

7. Zhang, M, Zhu, C-J: Global existence of solutions to a hyperbolic-parabolic system. Proc. Am. Math. Soc. 135(4), 1017-1027 (2007)

8. Li, T, Wang, Z-A: Nonlinear stability of traveling waves to a hyperbolic-parabolic system modeling chemotaxis. SIAM J. Appl. Math. 70(5), 1522-1541 (2009)

9. Li, T, Wang, Z-A: Asymptotic nonlinear stability of traveling waves to conservation laws arising from chemotaxis. J. Differ. Equ. 250(3), 1310-1333 (2009)

10. Corrias, L, Perthame, B, Zaag, H: A chemotaxis model motivated by angiogenesis. C. R. Math. Acad. Sci. Paris 336(2), 141-146 (2003)

11. Corrias, L, Perthame, B, Zaag, H: Global solutions of some chemotaxis and angiogenesis systems in high space dimensions. Milan J. Math. 72, 1-28 (2004)

12. Hao, C-C: Global well-posedness for a multidimensional chemotaxis model in critical Besov spaces. Z. Angew. Math. Phys. 63(5), 825-834 (2012)

13. Danchin, R: Global existence in critical spaces for compressible Navier-Stokes equations. Invent. Math. 141(3), 579-614 (2000)

14. Danchin, R: Fourier Analysis Methods for PDE's (2005); http://perso-math.univ-mlv.fr/users/danchin.raphael/ courschine.pdf

15. Ahmad, S, Le, D: Global and blow up solutions to cross diffusion systems. Adv. Nonlinear Anal. 4(3), $209-219$ (2015)

16. Li, H-L, Matsumura, A, Zhang, G-J: Optimal decay rate of the compressible Navier-Stokes-Poisson system in $R^{3}$. Arch. Ration. Mech. Anal. 196(2), 681-713 (2010)

17. Matsumura, A: An energy method for the equations of motion of compressible viscous and heat-conductive fluids. MRC Technical Summary Report, Univ. of Wisconsin-Madison, 2194 (1981)

18. Sohinger, V, Strain, R-M: The Boltzmann equation, Besov spaces, and optimal time decay rates in $R^{n}$. Adv. Math. 261 274-332 (2014)

19. Yu, H-J: Large time behavior of solutions for hyperbolic balance laws. J. Differ. Equ. 261(9), 4789-4824 (2016)

20. Majda, A: Compressible Fluid Flow and Systems of Conservation Laws in Several Space Variables. Applied Mathematical Sciences, vol. 53. Springer, New York (1984)

21. Ueda, Y, Wang, S, Kawashima, S: Dissipative structure of the regularity-loss type and time asymptotic decay of solutions for the Euler-Maxwell system. SIAM J. Math. Anal. 44(3), 2002-2017 (2012) 